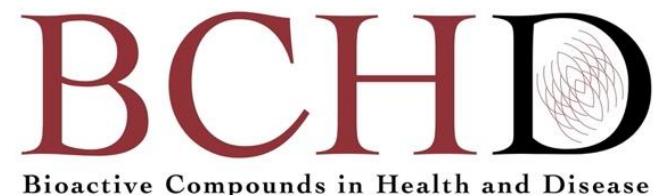

\title{
Increased Sirtuin expression, senescence regulating miRNAs, mtDNA, and bifidobacteria correlate with wellbeing and skin appearance after Sirtuin- activating drink
}

\section{Stephanie Lilja, Hanna Bäck, Carinna Stoll, Anna Mayer, Angelika Pointner, Berit Hippe, Ulrike Krammer, Alexander G. Haslberger*}

${ }^{1}$ Department of Nutritional Sciences, University of Vienna, 1090 Vienna, Austria

*Corresponding Author: Alexander Haslberger; Department of Nutritional Sciences, University of Vienna, 1090 Vienna, Austria

Submission Date: April 9 $9^{\text {th }}, 2021$; Acceptance Date: April 27 $7^{\text {th }}, 2021$; Publication Date: April $30^{\text {th }}, 2021$

Please cite this as: Lilja S., Bäck H., Stoll C., Mayer A., Pointner A., Hippe B., Krammer U., Haslberger A. Increased Sirtuin expression, senescence regulating miRNAs, mtDNA, and bifidobacteria correlate with wellbeing and skin appearance after Sirtuin- activating drink. Bioactive Compounds in Health and Disease 2021. 4(4): 45-62. DOI: https://www.doi.org/10.31989/bchd.v4i4.801

\section{ABSTRACT}

Background: Sirtuins attract high attention considering their properties to reverse molecular hallmarks of aging and age-related disorders. Many secondary plant ingredients (SPI) are known for their sirtuin-activating activities as well as epigenetic regulation of telomers, autophagy, senolysis, DNA repair but also improvement of gut microbiota. Furthermore, prebiotics enhanced butyrate and was shown to interact with SIRT pathways. This study investigated the effects of a drink containing a mix of different SPIs in combination with galactooligosaccharides (GOS) and their effect on SIRT activation, markers of aging relevant mechanisms, and gut microbiota composition in correlation with subjective wellbeing and skin structure appearance.
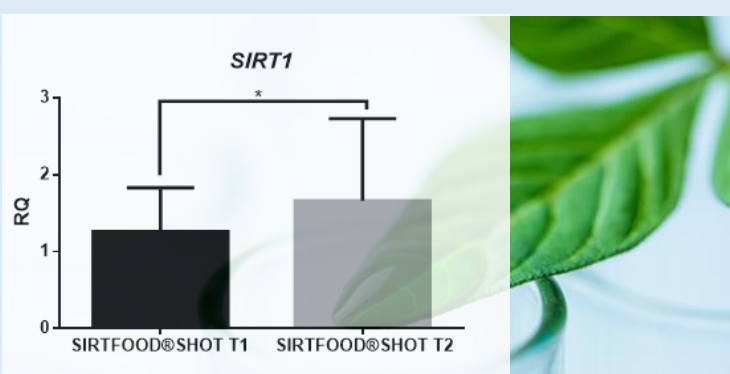

\section{secondary plant ingredients} and GOS containing drink :

- increased sirtuine expression

- Modulated gut microbiota compostion

- improved skin appearance and beauty aspects

-improved subjective well being 
Methods: We analyzed gene expression, mtDNA amount, and microbial composition in response to a sirtuinactivating drink in humans compared to a control group consuming a placebo. Food frequency, beauty, and general health questionnaires were asked, and a set of mRNAs and miRNAs were assessed using qPCR analysis. The gut composition was analyzed using Illumnia sequencing.

Results: SPI increased SIRT1, SIRT3, and modulated cell cycle relevant miR16 and senescence regulating miR34 expression. Additionally, mtDNA amount was higher in the group consuming the active supplement indicating an improved mitochondrial activity. The combined effect of SPI and GOS lead to an increase of Actinobacteria, especially Bifidobacterium, but also Veillonellaceae which was not observed in the control group. Significant correlations between SIRT3 expression and the gut microbiota Bifidobacterium and Veillonellaceae were observed. Additionally, statistical analysis of subjects self-reporting indicated beneficial effects regarding beauty and wellbeing.

Conclusion: Our results show that the combination of sirtuins inducing SPI and prebiotic GOS influences molecular pathways counteracting aging, senescence, inflammation, and enhanced groups of gut microbiota which are known to improve the innate and adaptive immune system.

Keywords: secondary plant ingredients, prebiotic, Sirtuins, subjective wellbeing, Bifidobacterium

CFFC 2020. This is an Open Access article distributed under the terms of the Creative Commons Attribution 4.0 License (http://creativecommons.org/licenses/by/4.0)

\section{INTRODUCTION}

Due to their regulatory roles in many pathophysiological conditions sirtuins (SIRTs) attract high attention. In mammals, seven SIRTs (SIRT1-7) have been identified and belong to the nicotinamide adenine dinucleotide (NAD+) dependent histone deacetylases (HDAC) [1-3]. HDAC removes the acetyl group from histones, which causes a condensed chromatin structure, thus results in gene silencing [1-2]. SIRT deacetylate histones and non-histone proteins and have been identified in distinct subcellular localization, thus being involved in multiple physiological processes, namely DNA repair, cell cycle regulation, energy metabolism, neuronal protection, gene expression regulation, and epigenetic activity [1][2][4]. SIRTs, located in the mitochondria are involved in amino- and fatty acid metabolism, oxidative phosphorylation, mtDNA replication, and regulation of anti-inflammatory agents [2-5].

Restrictive diets and their activation of SIRT1 have been intensively studied in vitro and in vivo, whereas the latter mostly in animal models than in humans [6]. Nevertheless, SIRT expression increases also in humans following Buchinger fasting guidelines [7]. During nutrition depletion, adenosine monophosphate (AMP) increases and adenosine triphosphate (ATP) decreases within the cell thus activating AMP-activated protein kinase (AMPK) and $\mathrm{NAD}+$, further activates SIRTs expression [8-9].

Secondary plant ingredients (SPI) are a broad range of bioactive botanicals and have been described to mimic this effect, thus defined as SIRT activating compounds (STACS). SPI can activate AMPK or SIRT directly or activating SIRT by targeting nicotinamide phosphoribosyltransferase (NAMPT), 
the enzyme in the NAD salvage pathway, which leads to an increased NAD+/NADH ratio [1][3][10]. Other studies show that polyphenols can target AMPK, by increasing the AMP/ATP ratio by interfering with the respiratory chain reaction in mitochondria, thus leading to activation of SIRTs [11]. SPI can also modulate micro RNAs (miRNA) leading to SIRT activation [12-13].

SPI which are defined by their low abundance have been used for centuries in traditional medicine due to their free radical scavenging action, antioxidative, antimicrobial and antiviral capacities. Polyphenolic compounds can therefore reduce cardiovascular risk due to low density lipoprotein oxidation inhibition [1][14][15]. Other SPIs exhibit anticancer, anti-obesity, cardio and neuroprotective effects and prevent type 2 diabetes by increasing insulin sensitivity [1].

Additionally, SPIs modulate gut microbiota (GM) [16]. Besides protecting against pathogens colonization, the GM protects integrity and permeability of the gut barrier and is part of the innate and adaptive immunoregulation. Moreover, the GM has several metabolic functions, such as synthesis of vitamins and short-chain fatty acids, absorption of dietary compounds, regulation of lipid, protein and carbohydrate metabolism and enzymatically modifies SPI bioavailability and healthy effect [16-17]. Age, diet, physical inactivity, stress and drugs are the main factors for GM dysbiosis and are linked to diseases regarding the gastrointestinal tract but also metabolic disorders, like obesity or diabetes [17].

SPIs and prebiotics favor the generation of short-chain fatty acids (SCFAs) and the SIRT expression in the gut mucosa. Both exert antiinflammatory effects, maintains gut barrier functions and beneficially effects clinical blood parameters
[18-20] but are also linked to be neuroprotective and regulate hormones, such as leptin, ghrelin, melatonin and serotonin, thus influencing hunger, satiety and mood [21-22]. Nevertheless, the range of short chain fatty acid (SCFAs) activities are limited comparing SPIs metabolites produced, within the gut[23]. Additionally, SPIs are usually found in combination in nature. Due to their heterogeneity, they exhibit different biologic activities and show synergistic properties [23].

Based on their multiple biological benefits and as potential STACs they are nowadays used in nutraceuticals or functional foods and in cosmetics to treat multiple (skin) aging signs [14][24][25]. We conducted a randomized, placebo-controlled, one blinded study to investigate if the SIRTFOOD ${ }^{\circledR}$ SHOT supplement, consisting of several potential natural SIRT activating compounds and SCFA producing prebiotic galactooligosaccharides (GOS) can address SIRTs expression in humans. Additionally, we observed the changes in the microbial gut composition, Food frequency questionnaire outcomes, regarding general health and beauty aspects and possible connections.

\section{MATERIALS AND METHODS}

Experimental design: 150 people were enrolled for the study performed at the University of Vienna, Department for nutritional science. The study was randomized, controlled, single-blinded and consists of two intervention groups: SIRTFOOD ${ }^{\circledR}$ SHOT supplementation (active) and placebo/control. The supplement was composed by the Swiss company System-Biologie AG (Wollerau). After dropouts, 100 participants attended the active group and 31 participants were consuming placebos, considering them as the control group. Dropouts were only seen in the intervention group, due to the lower 
tolerability of the bitter taste. The subjects were in average 41.12 years. The mean BMI was $26.0219 \pm$ $4.54906 \mathrm{~kg} / \mathrm{m}^{2}$ and weight $75.993 \pm 15.6306 \mathrm{~kg} .39$ participants $(29.8 \%)$ were male and 92 participants (70.2\%) were female. Exclusion criteria were participants taking pre- and probiotics, antibiotics, or sirtuin-activating compounds/medicine like metformin were excluded. Both groups were advised to keep their lifestyle and nutritional habits during the intervention. Only one flacon $(25 \mathrm{ml})$ of SIRTFOOD ${ }^{\circledR}$ SHOT or placebo had to be included during the day for three consecutive months. Following compounds can be found in one falcon (25ml) SIRTFOOD ${ }^{\circledR}$ SHOT: $3.5 \mathrm{mg}$ of gallic acid, $40 \mathrm{mg}$ EGCG, $25 \mathrm{mg}$ phloretin, $14 \mathrm{mg}$ anthocyanin, $10 \mathrm{mg}$ anthocyanidins, $6.4 \mathrm{mg}$ oleuropein and $0.9 \mathrm{mg}$ sulforaphane, $3 \mathrm{~g}$ galactooligosaccharides. The compounds were extracted from mango, apple, blueberry, olive leaves, broccoli, and green tea.

Sample collection and preparation: Food frequency questionnaires, general health and beauty questionnaires, dried blood spots, and stool samples were collected at two different time points at the beginning and after the intervention. In accordance with the declaration of the Viennese Human Ethics committee, all study participants gave written consent for the use of data. The beginning of the intervention is defined as time point 1 (T1) and T2 defines the end of the study.

Nutritional behavior and subjective well-being were validated via several standardized questionnaires. Total DNA and RNA were isolated from dried blood spots (DBS) using MagMAX FFPE DNA/RNA ultra-kit (Thermofisher, USA) via KingFisher Duo Prime purification system. Quantity and quality were assessed using a Nanodrop ND-1000 spectrophotometer (Nanodrop, Wilmington, DE,
USA). Relative mitochondrial DNA content was determined in genomic DNA isolated from the dried blood spots using a StepOne Plus real-time PCR Detection System (Applied Biosystem). For PCR, single-copy gene primers, mitochondrial primers (Biomers, Germany) and a LightCycler $^{\circledR} 480$ Sybr ${ }^{\circledR}$ Green I master mix (Roche) were used. cDNA was done eighter using LunaScript RT SuperMix Kit (Biolabs, Germany) for mRNA or TagMan Advanced miRNA cDNA synthesis kit for miRNAs. miRNA and mRNA of interest were determined using commercial primers (Thermofisher, USA) and TaqMan Fast advanced Mastermix (Thermofisher, USA) for RTqPCR amplifications performed on StepOne Plus realtime PCR Detection System (Applied Biosystem). Fold change was calculated using the formula of $2-\Delta \Delta \mathrm{Ct}$ $\left(\Delta \mathrm{Ct}=\mathrm{Ct}^{\text {gene of interest }}-\mathrm{Ct}^{\text {housekeeper }} ; \Delta \Delta \mathrm{Ct}=\Delta \mathrm{Ct}^{\mathrm{T2}}-\Delta \mathrm{Ct}^{\mathrm{T1}}\right.$ or $\Delta \Delta \mathrm{Ct}=\Delta \mathrm{Ct}-\Delta \mathrm{Ct}^{\mathrm{targt}}$ mean placebo control $)$ [26] [7]. Samples were generally run in $10 \mu \mathrm{L}$ reactions in doubles. Upon sample collection, stool samples were stored at $-80^{\circ} \mathrm{C}$. For sequencing microbial composition all fasting samples were analyzed by Biomes NGS GmbH (Germany) using Illumina Sequencing, written elsewhere [7] [27]

Statistical analysis: Raw microbial sequences were processed using the Quantitative Insights Into Microbial Ecology (QIIME) pipeline [28]. Taxonomy was assigned using the Ribosomal Database Project (RDP) classifier against Greengenes database. All data are presented as mean \pm standard deviation (SD). Data were analyzed using IBM SPSS Statistics for Windows Version 22.0 (IBM Corp., Armonk, NY, USA) and graph pad prism (Version 6). Paired t-test was used to compare the different time points for parametric and Wilcoxon test for nonparametric values. 


\section{RESULTS}

Population characteristics: The total study population size was 131 participants, divided into two different groups. The SIRTFOOD ${ }^{\circledR}$ SHOT group consists of 100 participants, consuming an active supplement, whereas 31 subjects were addressed as a control group, consuming the placebo shot. Males and females were almost normally contributed between the groups, although more females were interested to attend the study. Subjects were between 21 and 67 years, as well in the SIRTFOOD ${ }^{\circledR}$ SHOT as the placebo group. Although most characteristics were equal between the groups, participants in the control group had lower BMI (Table 1).

Table 1: Characteristics of the study population. Values were eighter given in total number $(\mathrm{N})$ and percentage or mean \pm standard deviation (SD)

\begin{tabular}{|c|c|c|c|}
\hline & \multicolumn{3}{|l|}{ study population } \\
\hline $\mathbf{N}$ & $131(100 \%)$ & $100(76.3 \%)$ & 31 (23.7\%) \\
\hline Male & 39 (29.8 \%) & $28(28 \%)$ & $11(35.5 \%)$ \\
\hline Female & $92(70.2 \%)$ & $72(72 \%)$ & $20(64.5 \%)$ \\
\hline \multicolumn{4}{|l|}{ Mean \pm SD } \\
\hline Age & $41.12 \pm 11.252$ & $42.11 \pm 11.267$ & $37.94 \pm 10.767$ \\
\hline Weight kg T1 & $75.993 \pm 15.6306$ & $76.915 \pm 16.6584$ & $72.871 \pm 11.5029$ \\
\hline BMI kg/ m² T1 & $26.0219 \pm 4.54906$ & $26.4248 \pm 4.84322$ & $24.7484 \pm 3.20067$ \\
\hline Weight kg T2 & $75.643 \pm 15.5512$ & $76.477 \pm 16.4663$ & $72.910 \pm 11.9174$ \\
\hline BMI kg/m² 2 & $25.9309 \pm 4.53196$ & $26.3211 \pm 4.73664$ & $24.6524 \pm 3.56280$ \\
\hline
\end{tabular}

Subjective well-being and beauty aspects: All participants were asked to interpret their skin appearance before and after the intervention. The placebo study population did not see any beneficial effects regarding beauty aspects. Study participants who consumed the active product had the impression of improved skin texture and its appearance. After the intervention period, $13 \%$ of the SIRTFOOD ${ }^{\circledR}$ SHOT population complained less about dry skin (Figure 1a). Moreover, $7.66 \%$ stated improved appearance of their skin (Figure 1b), including finer pores (9.63\%) (Figure 1c) and fewer wrinkles (3.65\%) (Figure 1d). At T1 $30 \%$ of the SIRTFOOD ${ }^{\circledR}$ SHOT population have stated to have brittle nails (Figure 1e). Thus $14.72 \%$ mentioned faster nail growth (Figure 1f), brittle nails deceased of $13 \%$ (Figure 1e). Moreover, $34 \%$ of the active group population stated a faster hair growth due to the intervention (Figure 1g), consequently, $21.9 \%$ reported less hair loss (figure $1 \mathrm{~h}$ ). General health aspects were collected of the total study population. After the intervention period, $38 \%$ of the SIRTFOOD ${ }^{\circledR}$ SHOT group reported to feel healthier (Figure $2 a$ ) and $43 \%$ felt more energy (Figure $2 b$ ), due to supplementation. Moreover, almost a quarter of the intervention group stated improved sleep (Figure 2c) and digestion (Figure 2d). 
a

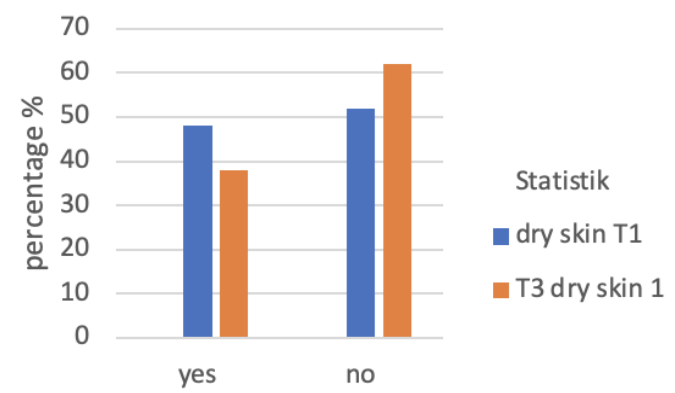

C

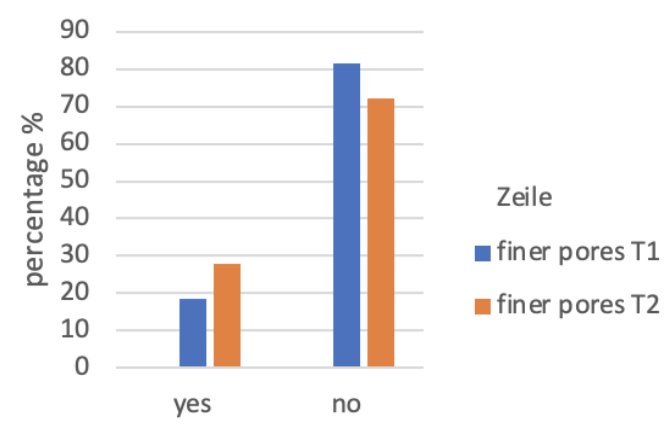

e
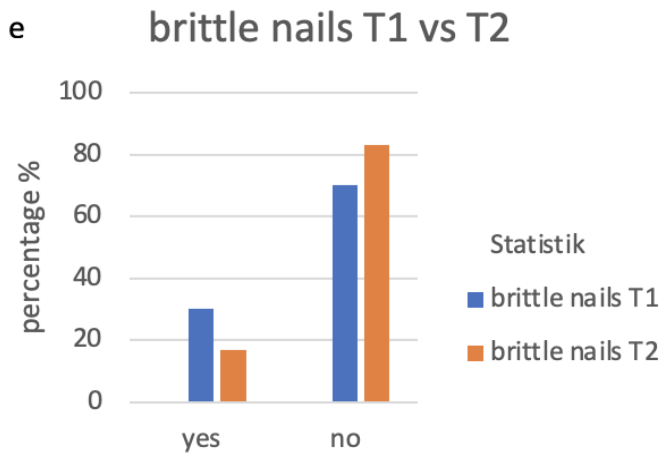

g faster hair gowth T2

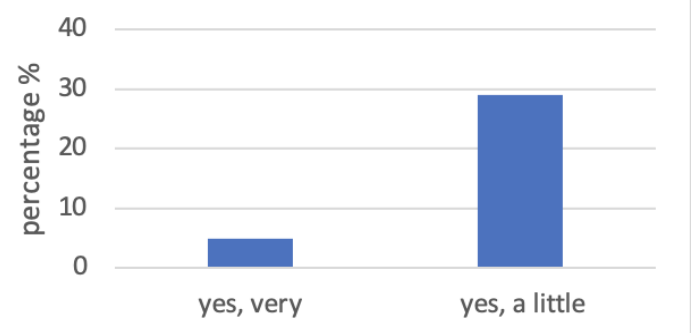

b appearance of skin improved T1 vs T2

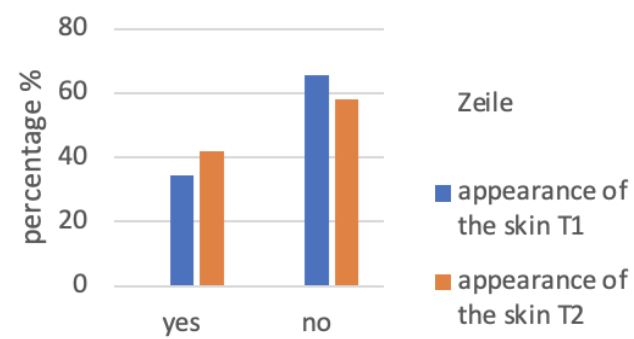

d impression of fewer wrinkles

T1 vs. T2

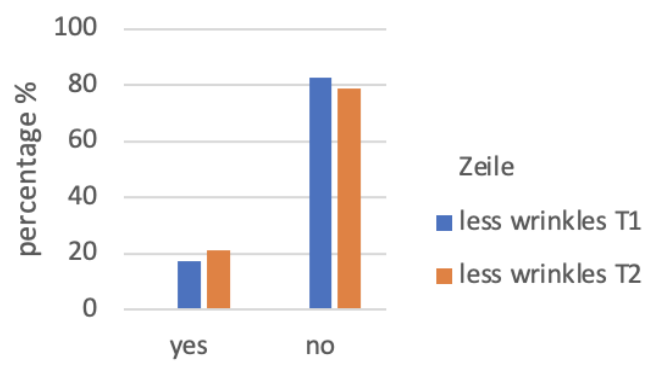

f fast nail growth T1 vs. T2

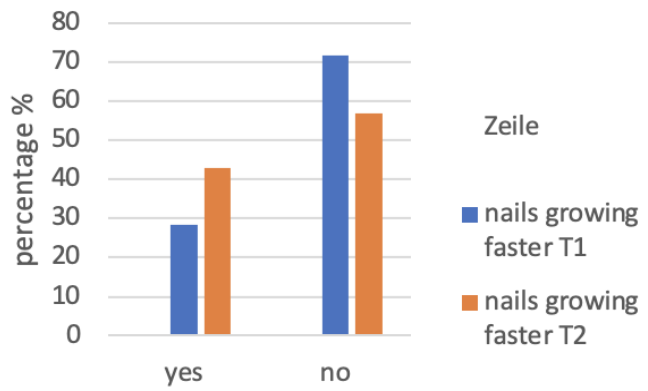

$\mathrm{h}$

less hair loss $\mathrm{T} 2$

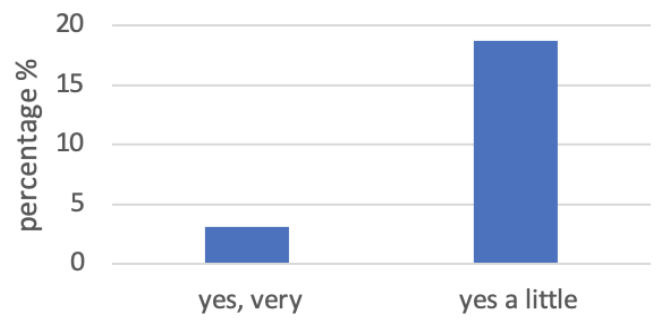

Figure 1. Analysis of questionnaire regarding beauty aspects. All results were given in percentage (\%) of total answers of the SIRTFOOS ${ }^{\circledR}$ SHOT group. Figure 1a- $f$ compared the answers of this study population between T1 and T2, whereas figure $1 \mathrm{~g}$ and $\mathrm{h}$ illustrates the results at the end of the timepoint. The impression of dry skin decreased between T1 and T2 (Figure 1a). After the intervention, 7.66\% more subjects consuming the active shot stated a better appearance of the skin (Figure 1b), including finer pores and fewer wrinkles (Figure 1c and 1d). Thus $14.72 \%$ mentioned faster nail growth (Figure 1f), brittle nails deceased of $13 \%$ (Figure 1e). $34 \%$ of the active group population stated a faster hair growth due to the intervention (Figure $1 \mathrm{~g}$ ), consequently, $21.9 \%$ reported less hair loss (figure $1 \mathrm{~h}$ ). 


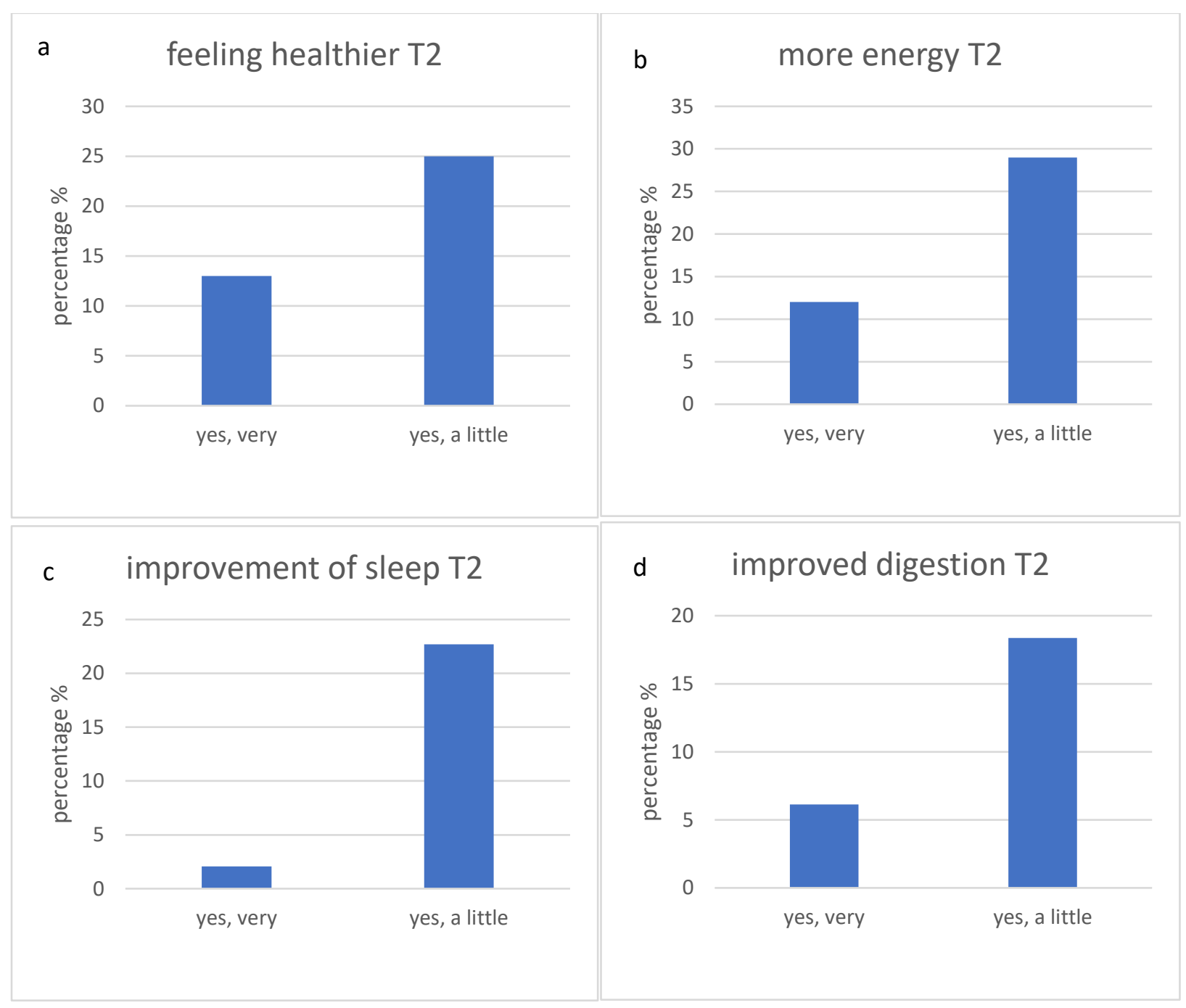

Figure 2. Analysis general health questionnaire. All results were given in percentage (\%) of total answers of the SIRTFOOS ${ }^{\circledR}$ SHOT group. 38\% reported to feel healthier (Figure $2 \mathrm{a}$ ) and $43 \%$ felt more energy after the intervention (Figure 2b). Moreover, almost a quarter of the intervention group stated improved sleep (Figure 2c) and digestion (Figure 2d), due to the supplementation.

Mitochondrial DNA content and gene expression: Higher mtDNA content could be detected in the SIRTFOOD ${ }^{\circledR}$ SHOT intervention compared to the control group, although only a statistical trend (Figure 3a). Moreover, changes in gene expression were seen after SIRTFOOD ${ }^{\circledR}$ SHOT supplementation. Lower miR34a- 5p (Figure 3b) and increased miR16-5p
(Figure 3c) expression were seen in the SIRTFOOD ${ }^{\circledR}$ SHOT group. Moreover, this study population showed different expression levels of MLH1, SIRT3 and SIRT1 compared to the control (figure 3d-f), Nevertheless, MLH1 and SIRT1 (Figure 3g) gene expression significantly increased and miR34a- $5 p$ significantly decreased after supplementation (data not shown). 

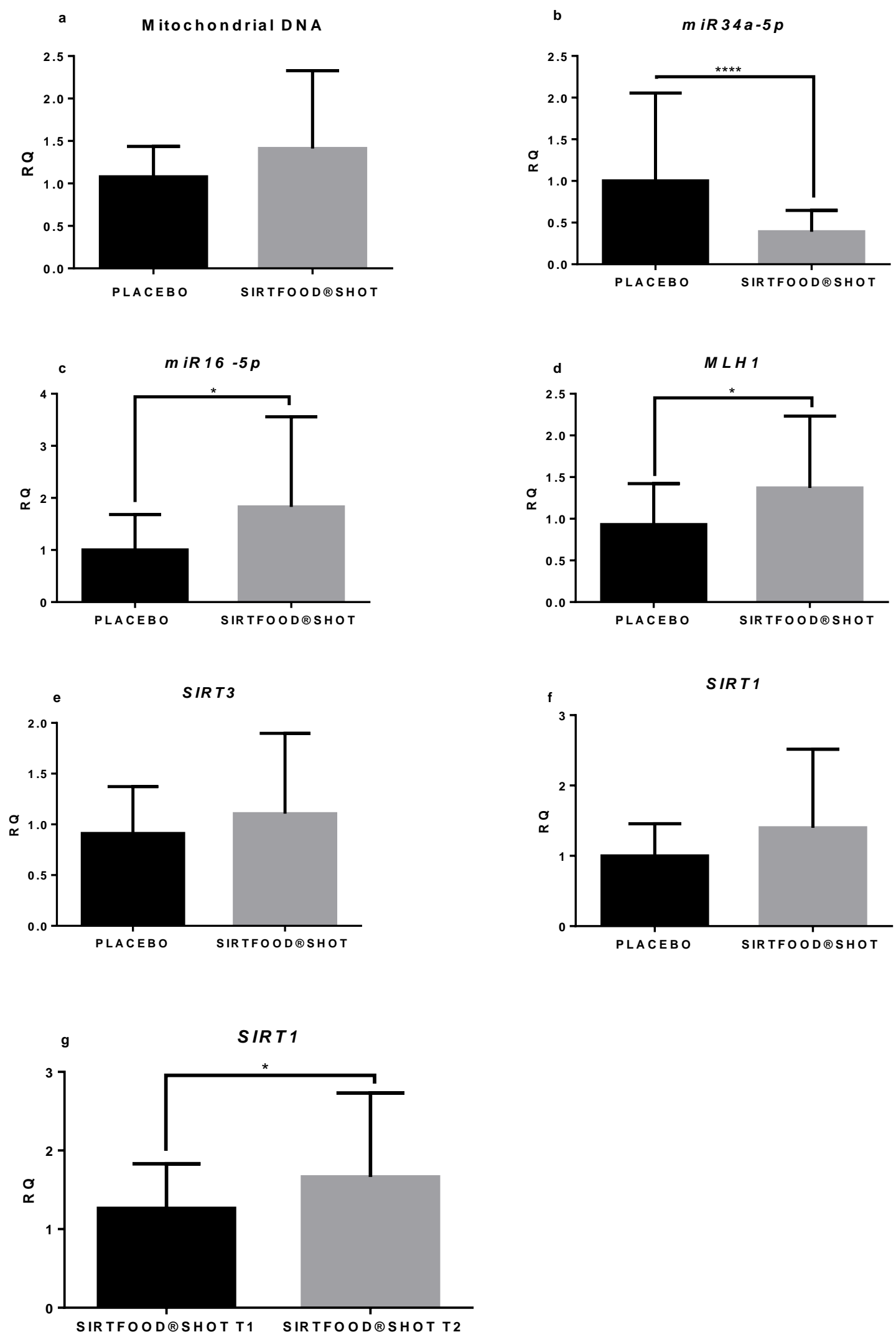

Figure 3: Results gene expression analysis. The results are expressed as mean +/- SD. Statistical significance between placebo and SIRTFOOD ${ }^{\circledR}$ SHOT (Figure 3a-f) and T1 and T2 of the SIRTFOOD ${ }^{\circledR}$ SHOT intervention (Figure 3g) was determined using paired t-test for parametric values and Wilcoxon test for nonparametric values. 
Gut microbiota composition: After SIRTFOOD ${ }^{\circledR}$ SHOT supplementation changes in the gut microbiota were observed. At the phylum level of Tenericutes, Lentisphaerae, Bacteroidetes, Euryarchaeota, Lentisphaerae and Actinobacteria increased, whereas only latter showed a strong statistical trend (Figure 4a). Decreased abundance of Cyanobacteria, Elusimicrobia, Proteobacteria, Verrucomicrobia, TM7 and Fusobacteria were observed after supplementation (Figure 4a). No changes were detected in Firmicutes abundance (Figure 4a). The same effects as observed in the Supplementation group were observed for Euryarchaeota, Lentisphaeae, Cyanobacteria, Elusimicrobia,
Proteobacteria, Verrucomicrobia and Firmicutes in the control group (Figure $4 \mathrm{~b}$ ). Contrary to the SIRTFOOD ${ }^{\circledR}$ SHOT group, no significant changes were seen in the abundance of Actinobacteria, Bacteroidetes, TM7. The amount of Tenericutes decreased (Figure 4b), whereas not statistically tested. In addition, at species level, no significant changes were detected for the placebo group (figure 4d), whereas Bifidobacterium increased in the intervention group, although only as a strong trend (figure 4c). Veillonellaceae increased for the SIRTFOOD ${ }^{\circledR}$ SHOT (Figure 4e) group but not for the placebo (Figure 4f). All SCFAs propionate, acetate and butyrate only increased slightly (data not shown).

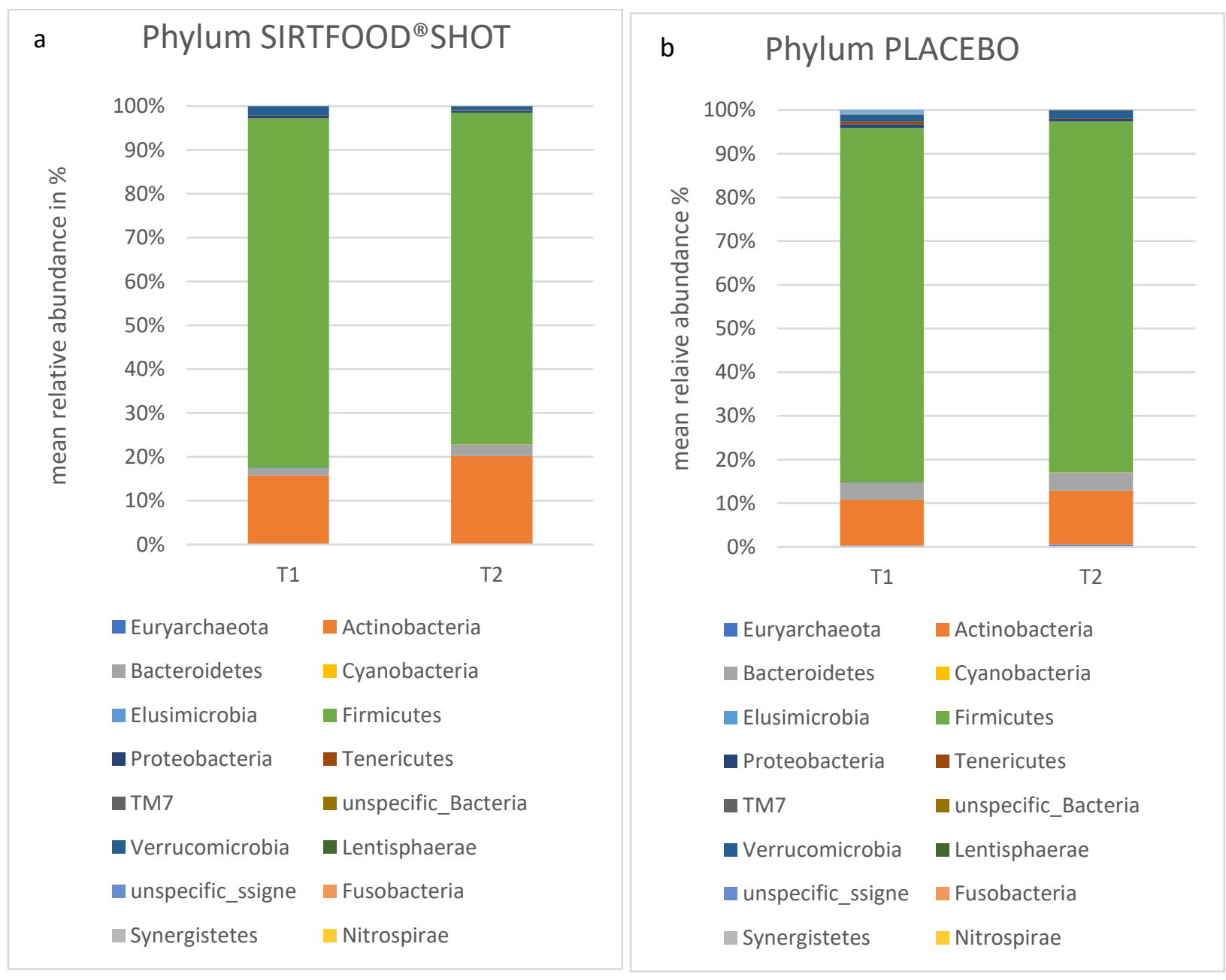


c

B ifid ob acterium

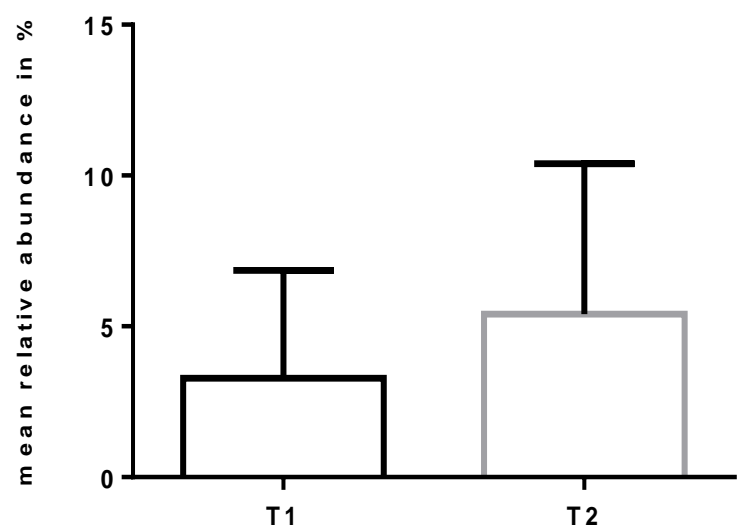

SIRTFOOD $\circledast S H O T$

e

Veillonellaceae

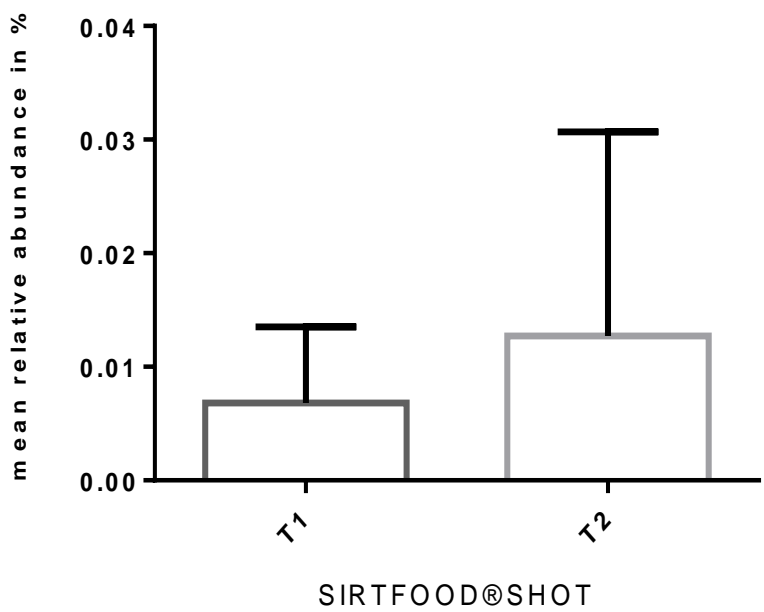

d

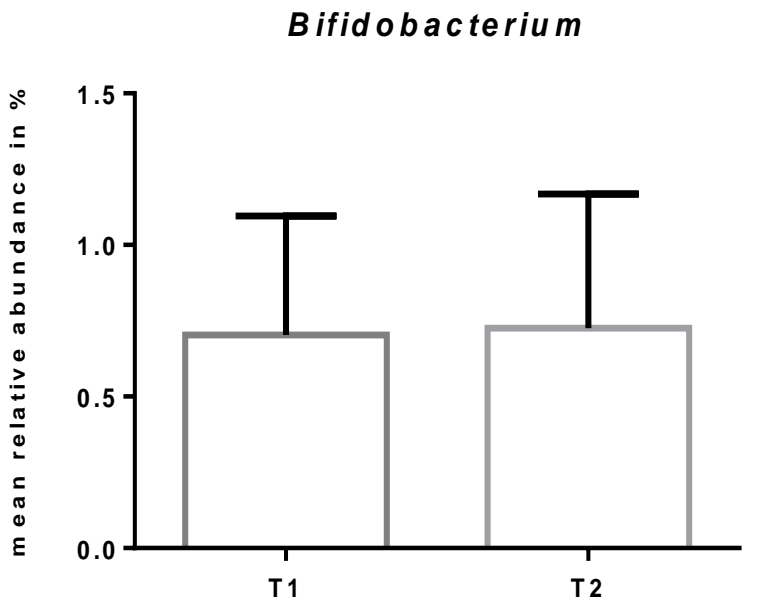

PLACEB O

f

Veillonellaceae

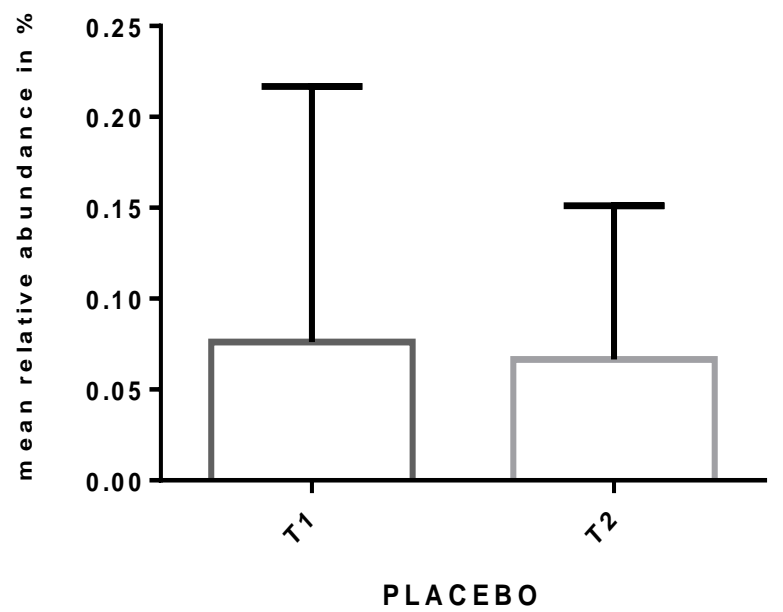

Figure 4. Microbial changes before and after treatment. Gut microbial changes before and after the treatment at phylum level showing Bifidobacterium and Veillonellaceae with the greatest change. Boxplot graphics demonstrating the elevation of Bifidobacterium before and after the intervention comparing to the placebo group, although only a trend $p=0.06$ for the SIRTFOOD ${ }^{\circledR}$ SHOT (c\&d). Veillonellaceae changes are demonstrated in figure e and f. The results are expressed as mean $+/$ SD. Statistical significance between T1 and T2 was determined using paired t-test for parametric values and Wilcoxon test for nonparametric values.

Correlations gut microbiota and gene expression:

Several correlations were obtained regarding gene expression and different gut microbiota members. MLH1 and Veillonellaceae, in addition SIRT3 and
Actinobacertia especially Bifidobacterium significant positive correlations were seen $(p<0.05)$ (Figure $5 a-c)$. Increasing SIRT3 negatively correlated with Bilophilia amount, although not statistically significant (Figure $5 d)$ 

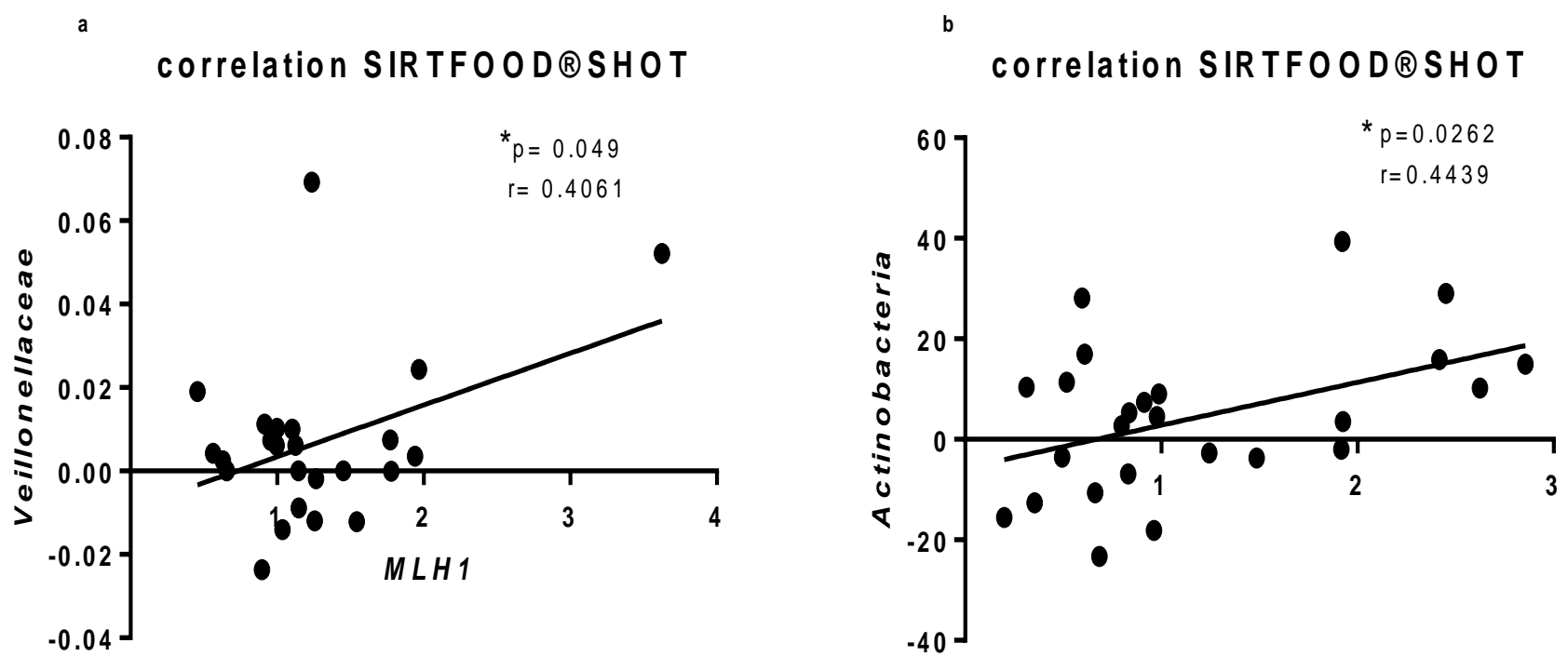

SIRT3

c correlation SIRTFOOD®SHOT
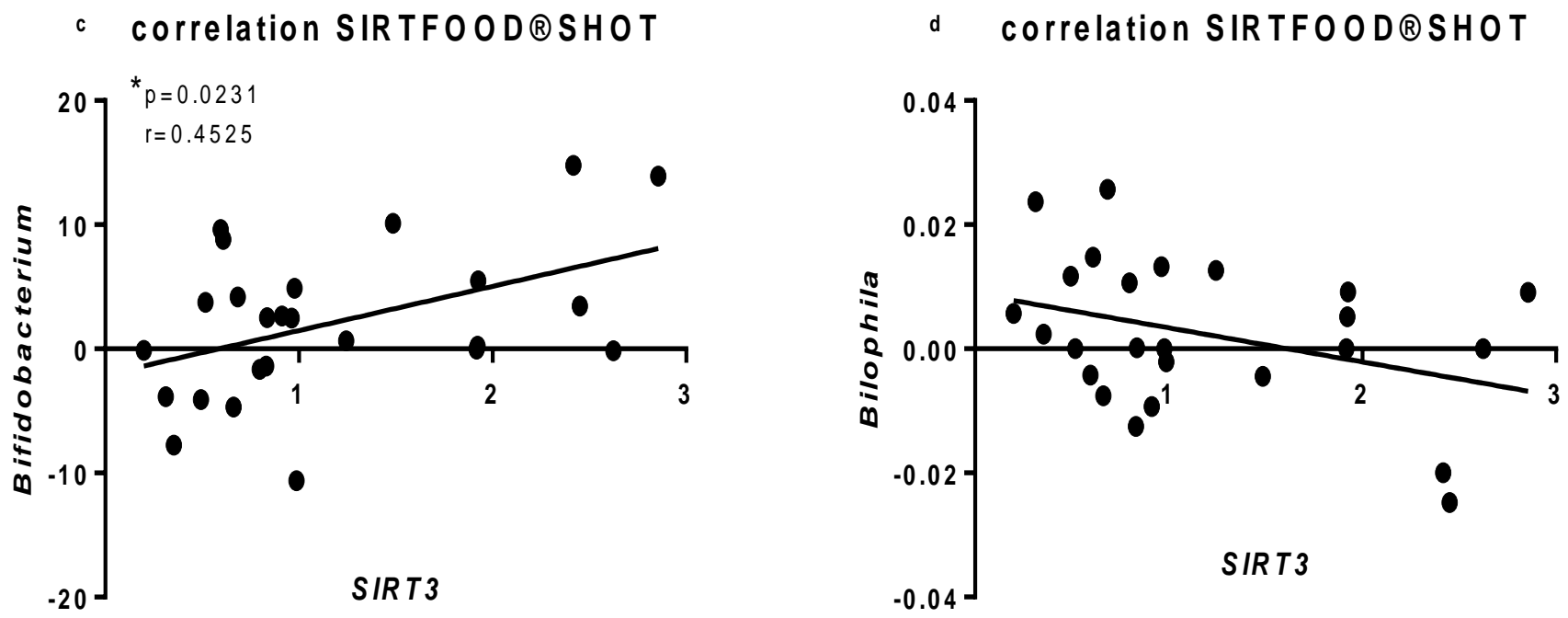

Figure 5: Pearson's correlations between microbiota and gene expression. a) positive correlation between $M L H I$ and Veillonellaceae ( $\mathrm{p}=0.049$ ). Positive correlation between SIRT3 and Actinobacteria (b), especially Bifidobacterium (c) $(\mathrm{p}<0.05)$. SIRT3 and Bilophilia negatively correlated, although only a trend $(d)(p=0.07)$. 


\section{DISCUSSION}

The human gut microbiota is a dynamic system that is influenced by its host lifestyle, genetics and epigenetics. The human genome and gut microbiome networking within metabolic pathways directly influence both normal physiology and disease processes[29]. SPIs and galactooligosaccharides exhibit prebiotic effects, which have beneficial immune, metabolic and cognitive functions [23]. The short chain fatty acids (SCFAs) produced by the gut microbiota are known to inhibit class I Histone deacetylases (HDACs) and also activate the UCP2AMPK-acetyl-CoA carboxylase (ACC) pathway with decreased $P P A R Y$ gene expression, thus resulting in decreased lipogenesis and increased AMP: ATP ratio. Latter induces AMP-activated protein kinase (AMPK) and sirtuins (SIRT) activation, having multiple beneficial effects on human health [23][30].

Improved digestion was reported by the participants after SIRTFOOD ${ }^{\circledR}$ SHOT supplementation. Kwon et al. investigated the effect of GM on defecation and concluded the increasing amount of Bifidobacterium is associated with increased stool frequency and better defecation [31]. This means increasing the amount of Bifidobacterium, which we observed after the active supplementation, might be helpful for people with obstipation.

Almost half of the participants from our study mentioned to feel healthier, having more energy, and better sleep after SIRTFOOD ${ }^{\circledR}$ SHOT treatment. Although, these results reflect subjective well-being, SPI and GM metabolites are known to exhibit antiinflammatory, cardio- and neuroprotective properties and influence metabolism [23]. Via methoxyindole pathway, the gastrointestinal tract is responsible for $95 \%$ of bodies serotonin synthesis from dietary tryptophan and is transported via the blood-brain barrier [23]. Because microbial composition is individual, metabolism and health effect can differ within individuals. Due to their prebiotic effect and modulation of GM, SPI supports tryptophan synthesis, the precursor of serotonin [23]. SPI favor the growth of beneficial GM, like Bifidobacterium, which we observed after SIRTFOOD ${ }^{\circledR}$ SHOT treatment but not in the control [17]. Bifidobacterium acts probiotic and Tian et al. [32] reported serotonin enhancement via modulating tryptophan biosynthesis pathways after Bifidobacterium supplementation [33][32]. Additionally, GM and its metabolites influence the sleep duration and quality of its host by influencing clock gene expression. Bifidobacterium has been found to improve the subject's sleep, both factors increased in our study [34]. Short sleep and bad quality are associated with GM dysbalance and counteract age-related diseases and increased mortality [34].

GM and its modulation by SPI play an important role in mood and gut health. Nevertheless, the host body is a fine-tuning network of many factors and epigenetic mechanisms play an essential role in gene expression thus human health. Thus, participants selfreported better well-being and sleep, this might also be explained by the increased miR16- $5 p$ expression in the SIRTFOOD ${ }^{\circledR}$ SHOT group compared to the placebo group. MiR16-5p can be modulated by SPIs [13][35] and regulates not only gut health by targeting claudin 2, which latter is predominantly expressed in leaky epithelia and upregulated in participants with bowel dysfunction [36]. Additionally, host miRNA can enter bacterial cells and regulate growth behavior and certain bacterial gene transcripts [37]. MiR16-5p, which decreases within age [38] is also an important regulator of the cell cycle, but also of serotonin thus regulating mood, 
satiety and sleep, factors protective against depression, eating disorders, and aging [39].

SPIs also impact miR34a- $5 p$, involved in the regulation of senescence. Additionally, overexpression is known to silence SIRT1 mRNA expression [40][41]. Obesity is associated with elevated miR34a $-5 p$ levels thus resulting in decreased SIRT1 expression. SIRT1 and SIRT3 enhance mitochondrial function, oxidative metabolism and counteract obesity [42]. Interestingly, the SIRTFOOD ${ }^{\circledR}$ SHOT group showed increased mitochondrial DNA amount compared to the control group. Weight loss (data not shown) was the same in both groups with an average $-0.5 \mathrm{~kg}$ after three months, thus more studies are needed to investigate sirtuins counteract obesity via weight loss in humans.

SIRT can also be expressed in human gut epithelial cells. Khalili et al. [19] reported a significant increase of SIRT1 expression after probiotic supplementation with Lactobacillus casei in participants with type 2 diabetes [19]. The same connections were seen in our study. A higher amount of SIRT3 expressed were seen with increased amount of Actinobacteria, especially Bifidobacterium. All three biomarkers were elevated after SIRTFOOD ${ }^{\circledR}$ SHOT treatment but not with the placebo. Interestingly, Chen et al [43] demonstrate, mice lacking SIRT3 leads to gut dysbiosis, intestinal permeability and inflammation following a high fat diet [43]. Additionally, Natividad et al. [44] reported increased amount of Bilophilia after high fat diet in mice, contributing to intestinal barrier dysfunction, inflammation, and metabolic syndrome. After SIRTFOOD ${ }^{\circledR}$ SHOT supplementation, we saw lower Bilophilia amount correlated with SIRT3 expression, implicating protective properties against metabolic syndrome.
Veillonellaceae increased in the active study population, but not in the control group. This specific group of microbiotas is known to metabolite lactate for energy production, a substrate contributing to fatigue during a physical performance and produced by Bifidobacterium [45][46]. Thus, an increase in the amount of Veillonellaceae may contribute to better physical performance, thus more energy, which the subjects reported. In addition, we observed a correlation between Veillonellaceae and MutL homolog 1 (MLH1). Latter belongs to DNA mismatch repairs and persons hypermethylated of one allele in MLH1, which contributes to lower MLH1 expression, having a predisposition to developing colorectal cancer [47] [48].

Veillonellaceae contribute to hosts adaptive immune system. Gut microbial dysbiosis can release toxins that induce human DNA damage, concurring its mutability, tumor induction and progression in gastrointestinal cancer [49]. EGCG, the polyphenol of green tea can reverse DNA damage [48]. Moreover, SPI can modulate the abundance of Veillonellaceae [50]

SIRTs are mostly known for their longevity related properties, which are all protecting against the hallmarks of aging [51][52] [4]. Based on this knowledge SIRTS are getting more attention to slowing down skin aging processes and develop cosmetics including natural STATs, although bioavailability and skin permeability are restricting factors for use of many potential active substances [25].

Premature skin aging is manifested by accelerated induction of wrinkling, scaling, roughness, dryness, laxity, and hyperpigmentation. After SIRTFOOD ${ }^{\circledR}$ SHOT supplementation up to $10 \%$ of the participants reported improved skin appearance, less dryness, finer pores and fewer wrinkles [53]. Even 
here the tight network between SPI, GM and Skin aging needs to be highlighted, thus an imbalance in GM leads to dermatological manifestations [54]. This communication is not only via the skin gut axis but also more directly via metastasis of $\mathrm{GM}$ and their metabolites in cases of disrupted intestinal barriers, which can be detected in patients' bloodstream [55] [29]. Besides being antioxidative and prebiotic, SPIs protect against skin aging via several mechanisms, including via SIRT activating, thus reducing inflammation and limit oxidative damage in tissues via SIRT3/SOD pathway in the mitochondria [56]. Additionally, SPI, like EGCG and resveratrol inhibit the collagen degradation enzyme MMP1, leading to wrinkles and tyrosinase, which leads to Hyperpigmentation [53][25].

More than one-third of the active gup reported faster hair and nail growth. Kubo et al. investigated the effect of several polyphenols regarding hair growth in mice. The results show resveratrol and fisetin as the strongest compound promoting hair growth mainly keeping hair follicles in the anagen phase via telomerase reverse transcription[57]. Several other studies investigated the hair growth potential of SPI, like cyanidins or EGCG [58][59] [60].

Many polyphenol-rich botanicals are considered to be adaptogenic: stress-modifying phytochemicals that increase organisms' nonspecific resistance to stress by increasing their ability to adapt and survive to external stressors and stimuli.

Thus, being one of the first studies investigating the possibility of SIRT activation by a combination of SPI and GOS in human and some outcomes reflect subjective wellbeing via FFQ this study needs to be considered as a pilot study and further interventions would be interesting to prove causality regarding specific mechanisms for a specific aspect.

\section{CONCLUSION}

Microbial dysbiosis or SIRT depletion leading to different pathologies, namely e.g. obesity, Alzheimer disease, Cancer, Diabetes type 2, Liver stenosis, depression, skin diseases. The results of the study demonstrating a broad range of beneficial effects in humans by supplementing a synergistic combination of prebiotics. Secondary plant ingredients not only elevate SIRT expression, it modulates gut microbiota and improved subjective wellbeing.

List of Abbreviation: SIRTs: sirtuins, STACs: sirtuinactivating compounds, SPI: secondary plant ingredients, mtDNA: mitochondrial DNA, NAD+: nicotinamide adenine dinucleotide, HDAC: histone deacetylases, AMP: adenosine monophosphate, ATP: adenosine triphosphate, NAMPT: nicotinamide phosphoribosyltransferase, miRNA: micro RNAs, GM: gut microbiota, SCFAs: short chain fatty acids, SD: standard deviation, MLH1: MutL homolog 1Author Contributions: S.L., A.H designed the research. S.L., H.B., C.S., conducted the research. S.L., H.B, T.D., A.M and B.H. performed clinical analysis. S.L., H.B., C.S. performed statistical analyses. S.L., A.P and A.H. wrote the manuscript. A.H. had primary responsibility for the final content. All authors read and approved the final version of the manuscript.

Declaration of competing interest: The authors declare that they have no known competing financial interests or personal relationships that could have appeared to influence their work in this paper. 
Acknowledgments and Funding: We thank the members of the University of Vienna; Department for Nutritional Science, and Biomes NGS GmbH for their assistance and support with the trial. The study was funded by grants of the Austrian research funding agency.

\section{REFERENCES}

1. Mayack BK, Sippl W, Ntie-Kang F: Natural products as modulators of sirtuins. Molecules 2020, 25(14):3287. DOI: $10.3390 /$ molecules 25143287.

2. Lee IH: Mechanisms and disease implications of sirtuinmediated autophagic regulation. Experimental \& Molecular Medicine 2019, 51(9):1-11. DOI: 10.1038/s12276-019-0302-7.

3. Bonkowski MS and Sinclair DA: HHS Public Access 2017, 17(11):679-690. DOI: 10.1038/nrm.2016.93.Slowing.

4. Lilja S, Oldenburg J, Pointner A, Dewald L, Lerch M, Hippe B, Switzeny O, et al.: Epigallocatechin Gallate Effectively Affects Senescence and Anti- SASP via SIRT3 in 3T3-L1 Preadipocytes in Comparison with Other Bioactive Substances. Oxidative Medicine and Cellular Longevity; 2020.

5. Li C and Zhou HM: The role of manganese superoxide dismutase in inflammation defense. Enzyme Research 2011, 2011:387176. DOI: 10.4061/2011/387176.

6. Wang Y: Molecular links between caloric restriction and Sir2/SIRT1 activation. Diabetes \& Metabolism Journal 2014, 38(5):321-329. DOI: 10.4093/dmj.2014.38.5.321.

7. Lilja S, Stoll C, Krammer U, Hippe B, Duszka K, Debebe T, Hofinger I, et al.: Five Days Periodic Fasting Elevates Levels of Longevity Related Christensenella and Sirtuin Expression in Humans. International Journal of Molecular Sciences 2021, 22(5):2331.

8. Newman JC and Verdin E: $\beta$-hydroxybutyrate: Much more than a metabolite. Diabetes Research and Clinical Practice 2014, 106(2):173-181. DOI: 10.1016/j.diabres.2014.08.009.

9. Bouwens $M$, Afman LA, Müller M: Fasting induces changes in peripheral blood mononuclear cell gene expression profiles related to increases in fatty acid $\beta$ oxidation: Functional role of peroxisome proliferatoractivated receptor $\alpha$ in human peripheral blood mononuclear cells. The American Journal of Clinical Nutrition 2007, 86(5):1515-1523. DOI: 10.1093/ajcn/86.5.1515.

10. Gertz M, Nguyen GTT, Fischer F, Suenkel B, Schlicker C, Fränzel B, Tomaschewski J, et al.: A Molecular Mechanism for Direct Sirtuin Activation by Resveratrol. PLOS One 2012, 7(11):1-12. DOI: 10.1371/journal.pone.0049761.

11. Zheng J and Ramirez VD: Inhibition of mitochondrial proton FOF1-ATPase / ATP synthase by polyphenolic phytochemicals. British Journal of Pharmacology 2000, 130(5):1115-1123.

12. Baker JR, Vuppusetty C, Colley T, Papaioannou Al, Fenwick $P$, Donnelly $L$, Ito $K$, et al.: Oxidative stress dependent microRNA-34a activation via PI3K $\alpha$ reduces the expression of sirtuin- 1 and sirtuin- 6 in epithelial cells. Scientific Reports 2016, 6:35871. DOI: 10.1038/srep35871.

13. Cione E, La Torre C, Cannataro R, Caroleo MC, Plastina P, Gallelli L: Quercetin, Epigallocatechin Gallate, Curcumin, and Resveratrol: From Dietary Sources to Human MicroRNA Modulation. Molecules 2020, 25(1):63.

14. Lavecchia T, Rea G, Antonacci A, Giardi MT: Healthy and Adverse Effects of Plant-Derived Functional Metabolites: The Need of Revealing their Content and Bioactivity in a Complex Food Matrix. Critical Reviews in Food Science and Nutrition 2013, 53(2):198-213. DOI: 10.1080/10408398.2010.520829.

15. Wink M: Modes of Action of Herbal Medicines and Plant Secondary Metabolites. Medicines 2015, 2(3):251-286. DOI: 10.3390/medicines2030251

16. Filosa S, Di Meo F, Crispi S: Polyphenols-gut microbiota interplay and brain neuromodulation. Neural Regeneration Research 2018, 13(12):2055-2059. DOI: 10.4103/1673-5374.241429.

17. Singh AK, Cabral C, Kumar R, Ganguly R, Rana HK, Gupta A, Lauro MR, et al.: Beneficial Effects of Dietary Polyphenols on Gut Microbiota and Strategies to Improve. Nutrients 2019, 11(9):2216.

18. Lakhan SE and Kirchgessner A: Gut microbiota and sirtuins in obesity-related inflammation and bowel dysfunction. Journal of Translational Medicine 2011, 9:202. DOI: 10.1186/1479-5876-9-202.

19. Khalili L, Alipour B, Jafar-Abadi MA, Faraji I, Hassanalilou T, Abbasi MM, Vaghef-Mehrabany E, et al.: The Effects of Lactobacillus casei on Glycemic Response, Serum Sirtuin1 and Fetuin-A Levels in Patients with Type 2 Diabetes 
Mellitus: A Randomized Controlled Trial. Iranian Biomedical Journal 2019, 23(1):68-77. DOI: 10.29252/ibj.23.1.68.

20. Harada G, Pattarawat $P$, Ito $K$, Matsumoto $T$, Hasegawa $T$, Katakura Y: Lactobacillus brevis T2102 suppresses the growth of colorectal cancer cells by activating SIRT1. Journal of Functional Foods 2016, 23:444-452. DOI: 10.1016/j.jff.2016.01.016.

21. Chandramowlishwaran P, Vijay A, Abraham D, Li G, Mwangi SM, Srinivasan S: Role of Sirtuins in Modulating Neurodegeneration of the Enteric Nervous System and Central Nervous System. Frontiers in Neuroscience 2020, 14(12):1-15. DOI: 10.3389/fnins.2020.614331.

22. Trebatická J and Ďuračková Z: Psychiatric Disorders and Polyphenols : Can They Be Helpful in Therapy? Oxidative Medicine and Cellualr Longevity 2015, 2015:248529.

23. Westfall S and Pasinetti GM: The Gut Microbiota Links Dietary Polyphenols With Management of Psychiatric Mood Disorders. Frontiers in Neuroscience 2019, 13(11):1-24. DOI: 10.3389/fnins.2019.01196.

24. Nizard C: Supplement The Science of Next Generation. no. February 2015, 2007.

25. Malinowska MA, Billet K, Douet S, Munsch T, Unlubayir M, Tungmunnithum D, Giglioli-Guivarc'h N, et al.: Grape Cane Extracts as Multifunctional Rejuvenating Cosmetic Ingredient: Evaluation of Sirtuin Activity, Tyrosinase Inhibition and Bioavailability Potential. Molecules 2020. 25(9):2203.

26. Duan $X$, Yang $Y$, Wang $S$, Feng $X$, Wang $T$, Wang $P$, Liu $S$, et al.: Changes in the expression of genes involved in cell cycle regulation and the relative telomere length in the process of canceration induced by omethoate. Tumor Biology 2017, 39(7):1-10. DOI: $10.1177 / 1010428317719782$.

27. Klindworth A, Pruesse E, Schweer T, Peplies J, Quast C, Horn M, Glöckner FO, et al.: Evaluation of general 16S ribosomal RNA gene PCR primers for classical and nextgeneration sequencing-based diversity studies. Nucleic Acids Research 2013, 41(1):1-11. DOI: $10.1093 / \mathrm{nar} / \mathrm{gks} 808$.

28. Caporaso JG, Kuczynskiet J, Stombaugh J, Bittinger K, Bushman FD, Costello EK, Fierer N, et al.: QIIME allows analysis of high- throughput community sequencing data. Nature Methods 2010, 7(5):335-336. DOI: 10.1038/nmeth0510-335.

29. Salem I, Ramser A, Isham N, and Ghannoum MA: The gut microbiome as a major regulator of the gut-skin axis. Front in Microbiology 2018, 9(7):1-14. DOI: 10.3389/fmicb.2018.01459.

30. Clark A and Mach N: The crosstalk between the gut microbiota and mitochondria during exercise. Frontiers in Physiology 2017, 8(5). DOI: 10.3389/fphys.2017.00319.

31. Kwon HJ, Lim JH, Kang D, Lim S, Park SJ, and Kim JH: Is stool frequency associated with the richness and community composition of gut microbiota? Intestistanl Research 2019, 17(3):419-426. DOI: 10.5217/ir.2018.00149.

32. Tian P, Wang G, Zhao J, Zhang H, Chen W: Bifidobacterium with the role of 5-hydroxytryptophan synthesis regulation alleviates the symptom of depression and related microbiota dysbiosis. Journal of Nutritioanl Biochemesitry 2019, 66:43-51. DOI: 10.1016/j.jnutbio.2019.01.007.

33. Cheng L-H, Liu Y-W, Wu C-C, Wang S, Tsai Y-C: Psychobiotics in mental health, neurodegenerativeand neurodevelopmental disorder. Journal of Food and Drug Analysis 2019, 27(3):632-648. DOI: https://doi.org/10.1016/j.jfda.2019.01.002.

34. Matenchuk BA, Mandhane PJ, Kozyrskyj AL: Sleep, circadian rhythm, and gut microbiota. Sleep Medicine Reviews 2020, 53:101340. DOI: 10.1016/j.smrv.2020.101340.

35. Dong Y, Chen H, Gao J, Liu Y, Li J, Wang J: Bioactive ingredients in Chinese herbal medicines that target noncoding RNAs: Promising new choices for disease treatment. Frontiers in Pharmacology 2019, 10(5):1-30. DOI: 10.3389/fphar.2019.00515.

36. C. Martínez, Rodiño-Janeiro BK, Lobo B, Stanifer ML, Klaus B, Granzow M, González-Castro AM, et al.: MiR-16 and miR-125b are involved in barrier function dysregulation through the modulation of claudin-2 and cingulin expression in the jejunum in IBS with diarrhoea. Gut 2017, 66(9):1597-1610. DOI: 10.1136/gutjnl-2016311477.

37. Hewel C, Kaiser J, Wierczeiko A, Linke J, Reinhardt C, Endres K, Gerber S: Common miRNA Patterns of Alzheimer's Disease and Parkinson's Disease and Their Putative Impact on Commensal Gut Microbiota. Frontiers in Neuroscience 2019, 13(3):1-13. DOI: 10.3389/fnins.2019.00113.

38. Tarallo S, Pardini B, Mancuso G, Rosa F, Di Gaetano, Rosina $\mathrm{F}$, Vineis $\mathrm{P}$, et al.: MicroRNA expression in relation 
to different dietary habits: A comparison in stool and plasma samples. Mutagenesis 2014, 29(5):385-391. DOI: 10.1093/mutage/geu028.

39. Tavares GA, Torres A, de Souza JA: Early Life Stress and the Onset of Obesity: Proof of MicroRNAs' Involvement Through Modulation of Serotonin and Dopamine Systems' Homeostasis. Frontiers in Physiology 2020, 11(7):1-8. DOI: 10.3389/fphys.2020.00925.

40. Filardi T, Sabato C, Lubrano C, Santangelo C, Morano S, Lenzi A, Migliaccio S, et al.: MicroRNA Modulation by Dietary Supplements in Obesity. Biomedicines 2020, 8(12):1-14. DOI: 10.3390/biomedicines8120545.

41. Torres LF, Cogliati B, and Otton R: Green Tea Prevents NAFLD by Modulation of miR-34a and miR-194 Expression in a High-Fat Diet Mouse Model. Oxidative Medicine and Cellular Longevity 2019, 2019. DOI: 10.1155/2019/4168380.

42. Sung-E C, Fu T, Seok S, Kim D-H, Yu E, Lee K-W, Kang Y, et al.: Elevated microRNA-34a in obesity reduces NAD+ levels and SIRT1 activity by directly targeting NAMPT. Aging Cell 2008, 28(30):4439-4448. DOI: 10.1111/acel.12135.Elevated.

43. Chen $M$, Hui S, Lang $H$, Zhou $M$, Zhang $Y$, Kang $C$, Zeng $X$, et al.: SIRT3 Deficiency Promotes High-Fat Diet-Induced Nonalcoholic Fatty Liver Disease in Correlation with Impaired Intestinal Permeability through Gut Microbial Dysbiosis. Molecular Nutrition \& Food Research 2019, 63(4):1-14. DOI: 10.1002/mnfr.201800612.

44. Natividad JM, Lamas B, Pham HP, Michel M-L, Rainteau D, Bridonneau C, da Costa G, et al.: Bilophila wadsworthia aggravates high fat diet induced metabolic dysfunctions in mice. Nature Communications 2018, 9(1):1-15. DOI: 10.1038/s41467-018-05249-7.

45. Pokusaeva K, Fitzgerald GF, and Van Sinderen D: Carbohydrate metabolism in Bifidobacteria. Genes \& Nutrition 2011, 6(3):285-306. DOI: 10.1007/s12263-0100206-6.

46. Scheiman J, Luber JM, Chavkin TA, MacDonald T, Tung A, Pham L-D, Wibowo MC, et al.: Meta-omics analysis of elite athletes identifies a performance-enhancing microbe that functions via lactate metabolism. Nature Medicine 2019, 25(7):1104-1109. DOI: 10.1038/s41591019-0485-4.

Hitchins MP, Wong JIL, Suthers G, Suter CM, Martin DIK, Hawkins NJ, Ward RL: Inheritance of a Cancer-Associated MLH1 Germ-Line Epimutation. The New England Journal of
Medicine 2007, 356:697-705.

47. Remely M, Ferk F, Sterneder S, Setayesh T, Roth S, Kepcija T, Noorizadeh R, et al.: EGCG Prevents High Fat DietInduced Changes in Gut Microbiota, Decreases of DNA Strand Breaks, and Changes in Expression and DNA Methylation of Dnmt1 and MLH1 in C57BL/6J Male Mice. Oxidative Medicine and Cellular Longevity 2017, 2017. DOI: $10.1155 / 2017 / 3079148$.

48. Docimo G, Cangiano A, Romano RM, Pignatelli MF, Offi C, Pagliconico VA, Galdiero M, et al.: The Human Microbiota in Endocrinology: Implications for Pathophysiology, Treatment, and Prognosis in Thyroid Diseases. Frontiers in Endocrinology (Lausanne) 2020, 11(12):4-10. DOI: 10.3389/fendo.2020.586529.

49. Loo YT, Howell K, Chan M, Zhang P, and Ng K: Modulation of the human gut microbiota by phenolics and phenolic fiber-rich foods. Comprehesive Reviews in Food Science and Food Safety 2020, 19(4):1268-1298. DOI: 10.1111/1541-4337.12563.

50. López-otín C, Blasco MA, Partridge L, Serrano M, and Kroemer G: The Hallmarks of Aging Longevity. Cell 2013, 153(6):1194-1217. DOI: 10.1016/j.cell.2013.05.039.The.

51. Hernandez-Segura A, Nehme J, and Demaria M: Hallmarks of Cellular Senescence. Trends in Cell Biology 2018, 28(6):436-453. DOI: 10.1016/j.tcb.2018.02.001.

52. Menaa F, Menaa A, and Tréton J: Polyphenols against Skin Aging. Polyphenols in Human Health and Disease 2013, 1:819-830. DOI: 10.1016/B978-0-12-3984562.00063-3.

53. Vaughn AR, Notay M, Clark AK, and Sivamani RK: Skin-gut axis: The relationship between intestinal bacteria and skin health. World Journal of Dermatology 2017, 6(4):5258. DOI: $10.5314 /$ wjd.v6.i4.52.

54. O'Neill CA, Monteleone G, McLaughlin JT, and Paus R: The gut-skin axis in health and disease: A paradigm with therapeutic implications. BioEssays 2016, 38(11):11671176. DOI: 10.1002/bies.201600008.

55. Ma C, Sun Y, Pi C, Wang H, Sun H, Yu X, Shi Y, et al.: Sirt3 Attenuates Oxidative Stress Damage and Rescues Cellular Senescence in Rat Bone Marrow Mesenchymal Stem Cells by Targeting Superoxide Dismutase 2. Frontiers in Cell Developmental Biology 2020, 8(11):1-13. DOI: 10.3389/fcell.2020.599376.

56. Kubo $C$, Ogawa $M$, Uehara $N$, and Katakura $Y$ : Fisetin Promotes Hair Growth by Augmenting TERT Expression. Frontiers in Cell and Developmental Biology 2020, 
8(10):1-13. DOI: 10.3389/fcell.2020.566617.

57. Kwon OS, Han JH, Yoo HG, Chung JH, Cho KH, Eun HC, Kim $\mathrm{KH}$, et al.: Human hair growth enhancement in vitro by green tea epigallocatechin-3-gallate (EGCG). Phytomedicine 2007, 14(7-8):551-555. DOI: 10.1016/j.phymed.2006.09.009.

58. Takahashi T, Kamiya T, Hasegawa A, Yokoo Y: Procyanidin oligomers selectively and intensively promote proliferation of mouse hair epithelial cells in vitro and activate hair follicle growth in vivo. Journal of Investigative Dermatology 1999, 112(3):310-316. DOI: 10.1046/j.1523-1747.1999.00532.x.

59. Tenore GC, Caruso D, Buonomo G, D'Avino M, Santamaria
$\mathrm{R}$, Irace C, Piccolo M, et al.: Annurca Apple Nutraceutical Formulation Enhances Keratin Expression in a Human Model of Skin and Promotes Hair Growth and Tropism in a Randomized Clinical Trial. Journal of Medicinal Food 2018, 21(1):90-103. DOI: 10.1089/jmf.2017.0016. 